J. Korean Math. Soc. 52 (2015), No. 4, pp. 781-795

http://dx.doi.org/10.4134/JKMS.2015.52.4.781

\title{
ON SEMI-ARMENDARIZ MATRIX RINGS
}

\author{
Kamil KozŁowski and Ryszard MaZureK
}

\begin{abstract}
Given a positive integer $n$, a ring $R$ is said to be $n$-semiArmendariz if whenever $f^{n}=0$ for a polynomial $f$ in one indeterminate over $R$, then the product (possibly with repetitions) of any $n$ coefficients of $f$ is equal to zero. A ring $R$ is said to be semi-Armendariz if $R$ is $n$-semi-Armendariz for every positive integer $n$. Semi-Armendariz rings are a generalization of Armendariz rings. We characterize when certain important matrix rings are $n$-semi-Armendariz, generalizing some results of Jeon, Lee and Ryu from their paper (J. Korean Math. Soc. 47 (2010), 719-733), and we answer a problem left open in that paper.
\end{abstract}

\section{Introduction}

Throughout this paper, all rings are associative, and all rings have an identity except where explicitly indicated. For a ring $R$, the ring of polynomials in the indeterminate $x$ over $R$ is denoted by $R[x]$, and if $A \subseteq R$, then $A[x]$ stands for the set of polynomials in $R[x]$ whose all coefficients belong to $A$.

Recall that a ring $R$ is said to be an Armendariz ring if whenever the product of two polynomials over $R$ is zero, then the products of their coefficients are all zero, that is, in the polynomial ring $R[x]$ the following holds:

$$
\begin{aligned}
& \text { for any } f=\sum_{i=0}^{k} a_{i} x^{i}, g=\sum_{j=0}^{m} b_{j} x^{j} \in R[x], \\
& \text { if } f g=0, \text { then } a_{i} b_{j}=0 \text { for all } i, j .
\end{aligned}
$$

The name for such rings was chosen to honor E. P. Armendariz, who noted in [2] that all reduced rings (i.e., rings containing no nonzero nilpotent elements) satisfy condition (1). Various interesting properties and constructions of Armendariz rings can be found, e.g., in [1], [4], [6], [8], [11], [13], [14] and [15].

Armendariz rings, as well as many other classes of Armendariz-like rings, have recently been objects of intensive investigation (see [13]). These new

Received September 12, 2014.

2010 Mathematics Subject Classification. 16N40, 16S36, 16S50.

Key words and phrases. $n$-semi-Armendariz ring, semi-Armendariz ring, upper triangular matrix ring. 
classes of Armendariz-like rings were defined using generalizations or modifications of condition (1). For example, by replacing in (1) the polynomial ring $R[x]$ with the power series ring $R[[x]]$, one obtains the definition of a powerserieswise Armendariz ring, introduced by N. K. Kim, K. H. Lee, and Y. Lee in [9]. By replacing in (1) the requirement that the products $a_{i} b_{j}$ are all zero with the condition that the products $a_{i} b_{j}$ are all nilpotent, we obtain the definition of a weak Armendariz ring, introduced by Z. Liu and R. Zhao in [12]. By considering in (1) the square of a single polynomial instead of the product of two polynomials, we obtain the definition of a 2-semi-Armendariz ring, introduced by Y. C. Jeon, Y. Lee and S. J. Ryu in [7], according to which a ring $R$ is said to be 2-semi-Armendariz provided for any polynomial $f=\sum_{i=0}^{m} a_{i} x^{i} \in R[x]$, if $f^{2}=0$, then $a_{i} a_{j}=0$ for all $i, j$.

More generally, for a positive integer $n$, in [7] Jeon, Lee and Ryu define a ring $R$ to be $n$-semi-Armendariz if for any polynomial $f=\sum_{i=0}^{m} a_{i} x^{i} \in R[x]$, $f^{n}=0$ implies $a_{i_{1}} a_{i_{2}} \cdots a_{i_{n}}=0$ for any subset $\left\{i_{1}, i_{2}, \ldots, i_{n}\right\} \subseteq\{0,1, \ldots, m\}$, and they call a ring $R$ a semi-Armendariz ring if $R$ is $n$-semi-Armendariz for every positive integer $n$. The following well-known result of D. D. Anderson and V. Camillo shows that all Armendariz rings are semi-Armendariz.

Proposition 1.1 ([1, Proposition 1]). Suppose $R$ is an Armendariz ring. If $f_{1}, f_{2}, \ldots, f_{n} \in R[x]$ are such that $f_{1} f_{2} \cdots f_{n}=0$, then $a_{1} a_{2} \cdots a_{n}=0$, where $a_{i}$ is a coefficient of $f_{i}$.

The following proposition summarizes basic properties of $n$-semi-Armendariz rings and semi-Armendariz rings.

Proposition 1.2 (see [7]).

(a) Every subring of an $n$-semi-Armendariz ring is $n$-semi-Armendariz.

(b) Direct sum of $n$-semi-Armendariz rings is n-semi-Armendariz.

(c) $A$ ring $R$ is $n$-semi-Armendariz if and only if the ring $R[x]$ is $n$-semiArmendariz.

(d) If a ring $R$ is semi-Armendariz, then $\operatorname{nil}(R[x]) \subseteq \operatorname{nil}(R)[x]$, where $\operatorname{nil}(A)$ denotes the set of nilpotent elements of a ring $A$.

The aim of this paper is to characterize when some important matrix rings are $n$-semi-Armendariz. The motivation for this work were results of Jeon, Lee and Ryu from their paper [7] and a problem left open in [7].

In [7, Theorem 1.2] it was proved that for any $n \geq 2$ the $n \times n$ upper triangular matrix ring $U_{n}(R)$ over a ring $R$ is $n$-semi-Armendariz if and only if $R$ is reduced. In Section 2 we extend this and some other results of [7] to the ring of upper triangular $n \times n$ matrices over a ring $R$ whose diagonal entries belong to a given subring $S$ of $R$ (see Proposition 2.4 and Theorem 2.5).

For a ring $R$ and an integer $n \geq 2$, the $\operatorname{ring} D_{n}(R)$ of upper triangular $n \times n$ matrices over $R$ whose diagonal entries are equal is a subring of $U_{n}(R)$. Hence it follows from the aforementioned result [7, Theorem 1.2] and Proposition 1.2(a) 
that if $R$ is a reduced ring, then the ring $D_{n}(R)$ is $n$-semi-Armendariz. It is natural to ask, whether the implication can be reversed, that is whether a ring $R$ has to be reduced if the ring $D_{n}(R)$ is $n$-semi-Armendariz. In [7] the problem was left unsolved. In Section 3 we show that for any integer $n \geq 2$ the answer to the problem is negative (see Example 3.2). The answer follows easily from a general result (Theorem 3.1), which also allows to construct further examples important for the theory of $n$-semi-Armendariz rings (see Examples 3.4 and $3.6)$.

In this paper, the full ring of $n \times n$ matrices over a ring $R$ is denoted by $M_{n}(R)$, and the ring of upper triangular $n \times n$ matrices over $R$ is denoted by $U_{n}(R)$. For a matrix $A \in M_{n}(R)$ and any $i, j \in\{1,2, \ldots, n\}$ the $(i, j)$ entry of $A$ is denoted by $A^{(i j)}$. The symbol $E_{i j}$ stands for the matrix with $(i, j)$ entry equal to 1 and all other entries equal to 0 (dimensions of the matrix $E_{i j}$ will be clear from the context). The canonical ring isomorphism of $M_{n}(R)[x]$ onto $M_{n}(R[x])$ is denoted by $\Phi$. Recall that the isomorphism $\Phi: M_{n}(R)[x] \rightarrow M_{n}(R[x])$ maps a polynomial

$$
f=A_{0}+A_{1} x+A_{2} x^{2}+\cdots+A_{k} x^{k} \in M_{n}(R)[x]
$$

to the $n \times n$ matrix $\Phi(f)$ over $R[x]$ whose $(i, j)$ entry is the polynomial

$$
A_{0}^{(i j)}+A_{1}^{(i j)} x+A_{2}^{(i j)} x^{2}+\cdots+A_{k}^{(i j)} x^{k} \in R[x]
$$

for all $i, j \in\{1,2, \ldots, n\}$. We will usually consider the isomorphism $\Phi$ restricted to a concrete subring of the $\operatorname{ring} M_{n}(R)$; such a restriction will still be denoted by $\Phi$.

\section{2. $n$-semi-Armendariz matrix rings}

The aim of this section is to identify $n$-semi-Armendariz subrings of the full matrix ring $M_{m}(R)$, where $R$ is a ring and $m \geq 2$. We start by showing that the ring $M_{m}(R)$ is never $n$-semi-Armendariz for $n \geq 2$.

Proposition 2.1. Let $R$ be a ring and let $m, n \geq 2$ be integers. Then the ring $M_{m}(R)$ is not n-semi-Armendariz.

Proof. Let $f=A_{0}+A_{1} x+A_{2} x^{2} \in M_{m}(R)[x]$, where

$$
A_{0}=E_{1 m}, A_{1}=E_{11}-E_{m m}, A_{2}=-E_{m 1} \text {. }
$$

Then $f^{2}=0$ and thus $f^{n}=0$. Since $A_{1}^{n}=E_{11}+(-1)^{n} E_{m m} \neq 0$, the ring $M_{m}(R)$ is not $n$-semi-Armendariz.

It is well known that for every $n \geq 2$ and arbitrary ring $R$, the upper triangular matrix ring $U_{n}(R)$ is not Armendariz (see [8, Example 1]). However, for any reduced ring $R$ the ring $U_{n}(R)$ is $n$-semi-Armendariz, which was proved in [7, Theorem 1.2] (and which shows that the class of $n$-semi-Armendariz rings is indeed wider than the class of Armendariz rings). In Theorem 2.5 below, we generalize this result by showing that for any reduced subring $S$ of an arbitrary ring $R$, the upper triangular $n \times n$ matrices over $R$ whose diagonal entries 
belong to $S$ form an $n$-semi-Armendariz ring. The following observation will be useful in our proofs.

Lemma 2.2. Let $R$ be a ring, let $I$ be an ideal of $R$ such that the factor ring $R / I$ is reduced, and let $m$ be a positive integer such that $I^{m}=0$. Then $R$ is $n$-semi-Armendariz for every $n \geq m$.

Proof. Clearly, the set $I[x]$ of polynomials from $R[x]$ with all coefficients in $I$ is an ideal of $R[x]$. Since the $\operatorname{ring} R / I$ is reduced, so is the ring $R[x] / I[x] \cong$ $(R / I)[x]$. Therefore, if a polynomial $f=a_{0}+a_{1} x+\cdots+a_{k} x^{k} \in R[x]$ satisfies $f^{n}=0$, then $f \in I[x]$ and thus $a_{i} \in I$ for any $i$. Hence, if $n \geq m$, then for any $i_{1}, i_{2}, \ldots, i_{n} \in\{0,1, \ldots, k\}$ we have $a_{i_{1}} a_{i_{2}} \cdots a_{i_{n}} \in I^{n} \subseteq I^{m}=0$, which proves that the ring $R$ is $n$-semi-Armendariz.

An immediate consequence of Lemma 2.2 is [7, Proposition 2.6]. Example 3.4 shows that the condition that $R / I$ is reduced in Lemma 2.2 is not superfluous.

Remark 2.3. Let $R$ be a ring. Recall that an ideal $J$ of $R$ is said to be completely prime if the factor ring $R / J$ is a domain. The intersection of all completely prime ideals of $R$ is called the generalized nil radical of $R$ and denoted by $\mathcal{N}_{g}(R)$ (see [3, Example 3.8.16]). Note that if $I$ is an ideal of $R$ such that the ring $R / I$ is reduced and $I^{m}=0$ for some positive integer $m$, then $I=\mathcal{N}_{g}(R)$. Indeed, since $I^{m}=0$, we deduce that $I$ is contained in every completely prime ideal of $R$ and $I \subseteq \mathcal{N}_{g}(R)$ follows. The opposite inclusion is an immediate consequence of the fact that any reduced ring is a subdirect product of domains (see [10, Theorem 12.7]). Thus $I=\mathcal{N}_{g}(R)$, as desired. Therefore, Lemma 2.2 can alternatively be formulated as follows: If $R$ is a ring such that $\mathcal{N}_{g}(R)^{m}=0$ for some positive integer $m$, then $R$ is $n$-semi-Armendariz for every $n \geq m$.

Let $S$ be a subring of a ring $R$. For any positive integer $n$ we set

$$
U_{n}(S, R)=\left\{A \in U_{n}(R) \mid A^{(i i)} \in S \text { for every } i \in\{1,2, \ldots, n\}\right\},
$$

i.e., $U_{n}(S, R)$ consists of all upper triangular $n \times n$ matrices over $R$ whose diagonal entries belong to $S$. Clearly, $U_{n}(S, R)$ is a subring of $U_{n}(R)$. Moreover, we set

$$
N_{n}(R)=\left\{A \in U_{n}(R) \mid A^{(i i)}=0 \text { for every } i \in\{1,2, \ldots, n\}\right\},
$$

i.e., $N_{n}(R)$ is the set of upper triangular $n \times n$ matrices over $R$ with all diagonal entries equal to zero. Obviously, $N_{n}(R)$ is an ideal of both $U_{n}(R)$ and $U_{n}(S, R)$.

The following result will be helpful in proving that some matrix rings of the form $U_{n}(S, R)$ are $k$-semi-Armendariz.

Proposition 2.4. Let $S$ be a subring of a ring $R$ and let $n$ be a positive integer. If there exists an ideal $I$ of $R$ such that $I \subseteq S$, and the ring $S / I$ is reduced, and $I^{m}=0$ for some positive integer $m$, then the ring $U_{n}(S, R)$ is $k$-semi-Armendariz for every $k \geq n+m-1$. 
Proof. Since $I$ is an ideal of $R$, the set

$$
J=\left\{A \in U_{n}(S, R) \mid A^{(i i)} \in I \text { for every } i \in\{1,2, \ldots, n\}\right\}
$$

is an ideal of $U_{n}(S, R)$. Furthermore, the factor $\operatorname{ring} U_{n}(S, R) / J$ is isomorphic to the direct sum of $n$ copies of the reduced ring $S / I$ and thus also $U_{n}(S, R) / J$ is reduced. Hence by Lemma 2.2, to complete the proof it suffices to show that $J^{n+m-1}=0$. For this, it is enough to show that

$$
\text { for any } A_{1}, A_{2}, \ldots, A_{n+m-1} \in J \text { we have } A_{1} A_{2} \cdots A_{n+m-1}=0 \text {. }
$$

Note that for any $i, j \in\{1,2, \ldots, n\}$ the $(i, j)$ entry of the matrix $A_{1} A_{2} \cdots A_{n+m-1}$ is a sum of products of the form

(2) $A_{1}^{\left(k_{1} k_{2}\right)} A_{2}^{\left(k_{2} k_{3}\right)} A_{3}^{\left(k_{3} k_{4}\right)} \cdots A_{n+m-1}^{\left(k_{n+m-1} k_{n+m}\right)}$, where $k_{1}=i$ and $k_{n+m}=j$.

If $k_{l}>k_{l+1}$ for some $l \in\{1,2, \ldots, n+m-1\}$, then $A_{l}^{\left(k_{l} k_{l+1}\right)}=0$ and thus the product (2) is equal to 0 in this case. Otherwise we have

$$
1 \leq i=k_{1} \leq k_{2} \leq k_{3} \leq \cdots \leq k_{n+m-1} \leq k_{n+m}=j \leq n .
$$

Hence in this case there must exist at least $m$ pairs $\left(k_{l}, k_{l+1}\right)$ with $k_{l}=k_{l+1}$, and since $A_{l} \in J$, for any such a pair $\left(k_{l}, k_{l+1}\right)$ we have $A_{l}^{\left(k_{l} k_{l+1}\right)} \in I$. Consequently, the product (2) belongs to $I^{m}$ and thus it is equal to zero. Therefore, $A_{1} A_{2} \cdots A_{n+m-1}=0$.

The following result extends [7, Theorem 1.2] to matrix rings of the form $U_{n}(S, R)$, with a different proof than that given in [7].

Theorem 2.5. Let $S$ be a subring of a ring $R$ and let $n \geq 2$ be an integer. Then

(a) The following conditions are equivalent:

(i) $U_{n}(S, R)$ is n-semi-Armendariz;

(ii) $U_{n}(S, R)$ is $k$-semi-Armendariz for every integer $k \geq n$;

(iii) $S$ is reduced.

(b) $U_{n+1}(S, R)$ is $n$-semi-Armendariz if and only if $S$ is reduced and $R$ is Armendariz.

Proof. (a) (i) $\Rightarrow$ (iii): Assume $U_{n}(S, R)$ is $n$-semi-Armendariz. Then by Proposition 1.2(a), the ring $U_{n}(S)$ is $n$-semi-Armendariz. Hence by [7, Theorem 1.2], the ring $S$ is reduced.

(iii) $\Rightarrow$ (ii): Apply Proposition 2.4 with $I=0$ and $m=1$.

(ii) $\Rightarrow$ (i): Obvious.

(b) We will use the following observation:

For any ring $T$ and matrices $B_{1}, B_{2}, \ldots, B_{n} \in N_{n+1}(T)$ we have

$$
B_{1} B_{2} \cdots B_{n}=B_{1}^{(1,2)} B_{2}^{(2,3)} \cdots B_{n}^{(n, n+1)} E_{1, n+1} .
$$


To establish (3), note that for any $i, j \in\{1,2, \ldots, n+1\}$ the $(i, j)$ entry of $B_{1} B_{2} \cdots B_{n}$ is a sum of products of the form

$$
B_{1}^{\left(k_{1} k_{2}\right)} B_{2}^{\left(k_{2} k_{3}\right)} B_{3}^{\left(k_{3} k_{4}\right)} \cdots B_{n}^{\left(k_{n} k_{n+1}\right)} \text {, where } k_{1}=i \text { and } k_{n+1}=j .
$$

For any $l \in\{1,2, \ldots, n\}$ we have $B_{l} \in N_{n+1}(T)$ and thus if $k_{l} \geq k_{l+1}$, then $B_{l}^{\left(k_{l} k_{l+1}\right)}=0$. Hence the product (4) can be nonzero only in the case when

$$
1 \leq i=k_{1}<k_{2}<k_{3}<\cdots<k_{n}<k_{n+1}=j \leq n+1
$$

that is, when $k_{l}=l$ for every $l \in\{1,2, \ldots, n+1\}$. Now (3) follows.

To prove (b), set $V=U_{n+1}(S, R)$ and $N=N_{n+1}(R)$. Assume $V$ is $n$-semiArmendariz. Since $U_{n}(S, R)$ is isomorphic to a subring of $V$, the $\operatorname{ring} U_{n}(S, R)$ is $n$-semi-Armendariz by Proposition $1.2(\mathrm{a})$, and thus by the already proved part (a), the ring $S$ is reduced. To show that $R$ is Armendariz, consider any polynomials $f=a_{0}+a_{1} x+\cdots+a_{k} x^{k}, g=b_{0}+b_{1} x+\cdots+b_{l} x^{l} \in R[x]$ such that $f g=0$. Without loss of generality we can assume that $k=l$. For any $m \in\{0,1, \ldots, k\}$ we set

$$
A_{m}=a_{m} E_{12}+b_{m} E_{23}+E_{34}+\cdots+E_{n, n+1} \in N,
$$

and we put

$$
h=A_{0}+A_{1} x+\cdots+A_{k} x^{k} \in N[x] .
$$

Let $\Phi: U_{n+1}(R)[x] \rightarrow U_{n+1}(R[x])$ be the canonical isomorphism, and let $H=$ $\Phi(h)$. Since $h \in N[x]$, it follows that $H \in N_{n+1}(R[x])$. Hence (3) implies

$H^{n}=H^{(1,2)} H^{(2,3)} H^{(3,4)} \cdots H^{(n, n+1)} E_{1, n+1}=f g\left(1+x+\cdots+x^{k}\right)^{n-2} E_{1, n+1}=0$, and thus $h^{n}=0$. Since $V$ is $n$-semi-Armendariz and $h^{n}=0$, it follows that $A_{i} A_{j} A_{1}^{n-2}=0$ for any $i, j$. Since $A_{i}, A_{j}, A_{1} \in N$, (3) implies

$$
a_{i} b_{j}=A_{i}^{(1,2)} A_{j}^{(2,3)} A_{1}^{(3,4)} \cdots A_{1}^{(n, n+1)}=0,
$$

which proves that $R$ is Armendariz.

To prove the converse, assume $S$ is reduced and $R$ is Armendariz, and consider any polynomial $q=A_{0}+A_{1} x+\cdots+A_{k} x^{k} \in V[x]$ with $q^{n}=0$. We claim that $A_{i} \in N$ for each $i$. To see this, note that since $S$ is reduced and $N$ is an ideal of $V$ such that $V / N$ is isomorphic to the direct sum of $n+1$ copies of $S$, the ring $V / N$ is reduced, and thus so is the $\operatorname{ring} V[x] / N[x] \cong(V / N)[x]$. Hence $q^{n}=0$ yields $q \in N[x]$, which proves our claim.

Let $\Phi: U_{n+1}(R)[x] \rightarrow U_{n+1}(R[x])$ be the canonical isomorphism, and let $Q=\Phi(q)$. Since $q \in N[x]$, it follows that $Q \in N_{n+1}(R[x])$. Furthermore, $Q^{n}=\Phi\left(q^{n}\right)=\Phi(0)=0$, and thus (3) implies

$$
Q^{(1,2)} Q^{(2,3)} \cdots Q^{(n, n+1)}=0 .
$$

Hence, if for any $i \in\{1,2, \ldots, n\}$ we set

$$
f_{i}=A_{0}^{(i, i+1)}+A_{1}^{(i, i+1)} x+\cdots+A_{k}^{(i, i+1)} x^{k} \in R[x],
$$


then $f_{1} f_{2} \cdots f_{n}=0$. Since $R$ is Armendariz, by Proposition 1.1 we have

$$
A_{s_{1}}^{(1,2)} A_{s_{2}}^{(2,3)} \cdots A_{s_{n}}^{(n, n+1)}=0 \text { for any } n \text {-tuple }\left(s_{1}, s_{2}, \ldots, s_{n}\right),
$$

and (3) implies $A_{s_{1}} A_{s_{2}} \cdots A_{s_{n}}=0$. Thus $V$ is $n$-semi-Armendariz.

As an immediate consequence of the above theorem we obtain [7, Theorem $1.2]$, which is just the first part of the following corollary. The second part says that if $R$ is a reduced ring, then $U_{n}(R)$ is a maximal $n$-semi-Armendariz subring of the full matrix $\operatorname{ring} M_{n}(R)$.

Corollary 2.6. For any ring $R$ and integer $n \geq 2$, the following conditions are equivalent:

(i) $U_{n}(R)$ is n-semi-Armendariz;

(ii) $U_{n}(R)$ is $k$-semi-Armendariz for every $k \geq n$;

(iii) $U_{n+1}(R)$ is $n$-semi-Armendariz;

(iv) $R$ is reduced.

If any of these equivalent conditions holds, then $U_{n}(R)$ is a maximal n-semiArmendariz subring of $M_{n}(R)$.

Proof. Since reduced rings are Armendariz, Theorem 2.5 implies that (i), (ii), (iii), and (iv) are equivalent. To prove the second part of the corollary, assume $R$ is reduced and $T$ is an $n$-semi-Armendariz subring of $M_{n}(R)$ such that $U_{n}(R) \varsubsetneqq T$. Then there exists a matrix $A \in T$ such that for some $i>j$ the $(i, j)$ entry of $A$, say $a$, is non-zero. Since $E_{i i}, E_{j j} \in T$, also $E_{i i} A E_{j j}=a E_{i j} \in T$. Thus for the matrices $A_{0}=a E_{i j}, A_{1}=a E_{i i}-a E_{j j}$ and $A_{2}=-a E_{j i}$ we have $f=A_{0}+A_{1} x+A_{2} x^{2} \in T[x]$. It is easy to see that $f^{2}=0$, and thus $f^{n}=0$. Since $T$ is $n$-semi-Armendariz, $0=A_{1}^{n}=a^{n} E_{i i}-a^{n} E_{j j}$. Hence $a^{n}=0$, and since $R$ is reduced, we obtain $a=0$, a contradiction.

The following result is an immediate consequence of Corollary 2.6.

Corollary 2.7 (cf. [7, Corollary 1.3]). For any ring $R$ the following conditions are equivalent:

(i) $R$ is reduced;

(ii) $U_{2}(R)$ is semi-Armendariz;

(iii) $U_{3}(R)$ is semi-Armendariz.

Given integers $n \geq 2$ and $m \geq n+2$, in the context of the first part of Corollary 2.6 it is natural to ask, when for a $\operatorname{ring} R$ the $\operatorname{ring} U_{m}(R)$ is $n$-semiArmendariz. The following result shows that the answer is "never" (cf. [7, Example 1.6]).

Proposition 2.8. Let $R$ be a ring and let $n \geq 2$ be an integer. Then for any integer $m \geq n+2$ the ring $U_{m}(R)$ is not $n$-semi-Armendariz.

The proof of Proposition 2.8 will be based on the following lemma. In the statement of the lemma, in an obvious way, we extend the notion of an $n$-semiArmendariz ring to rings without unity. Recall that $N_{n+2}(R)$ consists of upper 
triangular $(n+2) \times(n+2)$ matrices over $R$ with all diagonal entries equal to zero. Clearly, $N_{n+2}(R)$ is a non-unital subring of $U_{n+2}(R)$ (even more: it is an ideal of $\left.U_{n+2}(R)\right)$.

Lemma 2.9. For any ring $R$ and integer $n \geq 2$ the non-unital ring $N_{n+2}(R)$ is not n-semi-Armendariz.

Proof. We start the proof with the case $n=2$. Set $A=E_{12}+E_{34}$ and $B=E_{12}+E_{13}-E_{24}+E_{34}$. Then $f=A+B x \in N_{4}(R)[x]$ and $f^{2}=0$, but $A B=-E_{14} \neq 0$ and thus $N_{4}(R)$ is not 2-semi-Armendariz.

Next assume that $n \geq 3$. In this case we simply repeat arguments of $[7$, Example 1.6]. We set

$$
\begin{gathered}
A=E_{12}+E_{23}+\cdots+E_{n-2, n-1}+E_{n-1, n+1}+E_{n, n+2} \in N_{n+2}(R), \\
B=E_{n-1, n}+E_{n-1, n+1}+E_{n, n+2}-E_{n+1, n+2} \in N_{n+2}(R) .
\end{gathered}
$$

It is easy to verify that $B^{2}=B A^{2}=B A B=0$,

$$
A^{k}=E_{1, k+1}+E_{2, k+2}+\cdots+E_{n-k-1, n-1}+E_{n-k, n+1} \text { for } 2 \leq k \leq n-2,
$$

and $A^{n-1}=E_{1, n+1}$. Hence for $f=A+B x \in N_{n+2}(R)[x]$ we have

$$
f^{n}=A^{n}+\left(A^{n-2} B A+A^{n-1} B\right) x=\left(E_{1, n+2}-E_{1, n+2}\right) x=0 .
$$

Since $A^{n-2} B A=E_{1, n+2} \neq 0$, the ring $N_{n+2}(R)$ is not $n$-semi-Armendariz.

Now we are ready to prove Proposition 2.8.

Proof of Proposition 2.8. From Proposition 1.2(a) and Lemma 2.9 we deduce that the ring $U_{n+2}(R)$ is not $n$-semi-Armendariz. To complete the proof, consider any integer $m \geq n+2$. Then the $\operatorname{ring} U_{n+2}(R)$ is isomorphic to a subring of $U_{m}(R)$. Since the ring $U_{n+2}(R)$ is not $n$-semi-Armendariz, Proposition 1.2(a) implies that $U_{m}(R)$ is not $n$-semi-Armendariz.

Let $R$ be a ring and let $n, k$ be integers such that $2 \leq n<k$. By Corollary 2.6, $R$ being reduced implies that the ring $U_{n}(R)$ is $k$-semi-Armendariz. The following example shows that the opposite implication is not true.

Example 2.10. (For any $2 \leq n<k$ there exists a nonreduced ring $R$ such that the ring $U_{n}(R)$ is $k$-semi-Armendariz.) Let $p$ be a prime number, and let $m=k-n+1$. Let $R=\mathbb{Z}_{p^{m}}$ be the ring of integers modulo $p^{m}$ and let $I=(p)$ be the ideal of $R$ generated by $p$. Then the factor $\operatorname{ring} R / I \cong \mathbb{Z}_{p}$ is reduced and $I^{m}=0$. Hence by Proposition 2.4 the ring $U_{n}(R)$ is $k$-semi-Armendariz. Furthermore, since $m \geq 2$, the ring $R$ is not reduced.

Corollary 2.6 implies that the ring $U_{n}(R)$ constructed in Example 2.10 is not $n$-semi-Armendariz. Hence, the same example shows also that for any $2 \leq n<k$ there exists a $k$-semi-Armendariz ring that is not $n$-semi-Armendariz (cf. [7, p. 725]). 


\section{Answer to Jeon-Lee-Ryu's problem}

Given an integer $n \geq 2$, in [7, Theorem 1.2] Jeon, Lee and Ryu characterized rings $R$ for which the ring $U_{n}(R)$ is $n$-semi-Armendariz. Namely, they proved that $U_{n}(R)$ is $n$-semi-Armendariz if and only if $R$ is reduced (see also Corollary $2.6)$. Since the set

$$
D_{n}(R)=\left\{A \in U_{n}(R) \mid A^{(1,1)}=A^{(2,2)}=\cdots=A^{(n, n)}\right\}
$$

(i.e., the set of all upper triangular $n \times n$ matrices over $R$ whose diagonal entries are equal) is a subring of $U_{n}(R)$, it follows from Proposition 1.2(a) that if $R$ is a reduced ring, then the ring $D_{n}(R)$ is n-semi-Armendariz. It is natural to ask, whether the implication can be reversed, which led the authors of [7] to the following problem (see [7, p. 724, the paragraph preceding Proposition 1.5]):

(5) Problem: If $n \geq 2$ and the ring $D_{n}(R)$ is $n$-semi-Armendariz, must $R$ be a reduced ring?

In [7] the problem was left unsolved. In Example 3.2 we will show that the answer to the problem is negative for any integer $n \geq 2$. The answer will easily follow from the general Theorem 3.1.

The main reason why in Theorem 3.1 we focus on rings $D_{n}(S)$ with $S$ of the form $S=D_{2}(R)$ is that the $\operatorname{ring} S=D_{2}(R)$ is never reduced, so in the context of Jeon-Lee-Ryu's problem (5) it is natural to ask, when the ring $D_{n}(S)=D_{n}\left(D_{2}(R)\right)$ is $n$-semi-Armendariz. In the theorem below we answer this question in the case where the ring $R$ is reduced and commutative.

For a ring $R$, if $n$ is a positive integer and $r \in R$, then $n r$ denotes the sum $r+r+\cdots+r$ with $n$ summands.

Theorem 3.1. Let $R$ be a reduced commutative ring and let $n$ be a positive integer. Then the following conditions are equivalent:

(i) The ring $D_{n}\left(D_{2}(R)\right)$ is n-semi-Armendariz;

(ii) The ring $\left(D_{2}(R)[x]\right) /\left(x^{n}\right)$ is $n$-semi-Armendariz, where $\left(x^{n}\right)$ denotes the ideal of $D_{2}(R)[x]$ generated by $x^{n}$;

(iii) $n r=0 \Rightarrow r=0$ for any $r \in R$.

Proof. The case $n=1$ is trivial, because every ring is obviously 1 -semi-Armendariz. Thus to the end of the proof we assume that $n \geq 2$.

(i) $\Rightarrow$ (ii): Assume the ring $D_{n}\left(D_{2}(R)\right)$ is $n$-semi-Armendariz. The factor ring $\left(D_{2}(R)[x]\right) /\left(x^{n}\right)$ is isomorphic to the matrix ring

$$
\left\{\left(\begin{array}{ccccc}
A_{1} & A_{2} & \cdots & A_{n-1} & A_{n} \\
0 & A_{1} & \ddots & & A_{n-1} \\
\vdots & \ddots & \ddots & \ddots & \vdots \\
0 & 0 & \ddots & A_{1} & A_{2} \\
0 & 0 & \cdots & 0 & A_{1}
\end{array}\right) \mid A_{1}, A_{2}, \ldots, A_{n} \in D_{2}(R)\right\}
$$


which is a subring of $D_{n}\left(D_{2}(R)\right)$. Hence Proposition 1.2(a) implies that the ring $\left(D_{2}(R)[x]\right) /\left(x^{n}\right)$ is $n$-semi-Armendariz.

(ii) $\Rightarrow$ (iii): Put $P=\left(D_{2}(R)[x]\right) /\left(x^{n}\right)$ and assume $P$ is $n$-semi-Armendariz. We will use the bar notation for elements of $P$, that is, for any $h \in D_{2}(R)[x]$ we will write $\bar{h}$ to denote the coset $h+\left(x^{n}\right) \in P$. Let $r \in R$ be such that $n r=0$. Set

$$
A=\left(\begin{array}{ll}
r & 0 \\
0 & r
\end{array}\right), B=\left(\begin{array}{ll}
0 & 1 \\
0 & 0
\end{array}\right) \in D_{2}(R)
$$

and consider the polynomial $f=\bar{A} \bar{x}+\bar{B} t \in P[t]$. Note that in $P$ we have

$$
\bar{A} \bar{B}=\bar{B} \bar{A}, \bar{B}^{2}=0, \bar{x}^{n}=0 \text {, and } n \bar{A}=0 \text {. }
$$

Hence, by the binomial theorem,

$$
f^{n}=(\bar{A} \bar{x}+\bar{B} t)^{n}=\sum_{i=0}^{n}\left(\begin{array}{c}
n \\
i
\end{array}\right)(\bar{A} \bar{x})^{n-i}(\bar{B} t)^{i}=\bar{A}^{n} \bar{x}^{n}+n \bar{A}^{n-1} \bar{x}^{n-1} \bar{B} t=0 .
$$

Since the ring $P$ is $n$-semi-Armendariz, it follows that $(\bar{A} \bar{x})^{n-1} \bar{B}=0$ and thus $A^{n-1} B x^{n-1} \in\left(x^{n}\right)$. Hence $A^{n-1} B=0$ and consequently $r^{n-1}=0$. Since $R$ is reduced, we obtain $r=0$, as desired.

(iii) $\Rightarrow$ (i): Set $D(R)=D_{n}\left(D_{2}(R)\right)$. To the end of the proof the ring $D(R)$ is considered as a subring of $U_{2 n}(R)$. For any $B \in D(R)$ and $i, j \in\{1,2, \ldots, 2 n\}$, $B^{(i j)}$ denotes the $(i, j)$ entry of the matrix $B$.

Obviously $N(R)=\left\{B \in D(R): B^{(i i)}=0\right.$ for any $\left.i \in\{1,2, \ldots, 2 n\}\right\}$ is an ideal of $D(R)$. We start with describing the form of any product of $n$ matrices from $N(R)$, which will be crucial in the proof of the implication (iii) $\Rightarrow$ (i). The reader should note that in this part of the proof we do not put any assumption on the ring $R$.

Let $B_{1}, B_{2}, \ldots, B_{n} \in N(R)$ and let $B=B_{1} B_{2} \cdots B_{n}$. Then for any $i, j \in$ $\{1,2, \ldots, 2 n\}$, the $(i, j)$ entry of $B$ is a sum of products of the form

(6) $\quad b=B_{1}^{\left(k_{0} k_{1}\right)} B_{2}^{\left(k_{1} k_{2}\right)} B_{3}^{\left(k_{2} k_{3}\right)} \cdots B_{n}^{\left(k_{n-1} k_{n}\right)}$, where $k_{0}=i$ and $k_{n}=j$.

Assume $b \neq 0$. Then $B_{t}^{\left(k_{t} k_{t+1}\right)} \neq 0$ for every $t \in\{0,1, \ldots, n-1\}$, and since $B_{t} \in N(R)$, it follows that $k_{t}<k_{t+1}$. Thus

$$
1 \leq i=k_{0}<k_{1}<k_{2}<\cdots<k_{n-1}<k_{n}=j \leq 2 n .
$$

Notice that for any matrix $A \in D(R)=D_{n}\left(D_{2}(R)\right)$ we have $A^{(p q)}=0$ for every pair $(p, q)$ with even $p$ and odd $q$. Hence, since $b \neq 0$, if $k_{t}$ is even for some $t \in\{0,1, \ldots, n-1\}$, then so are $k_{t+1}, k_{t+2}, \ldots, k_{n}$. In particular, if $k_{0}$ would be even, then so would be $k_{1}, k_{2}, \ldots, k_{n}$, and we would obtain $n+1$ even integers in the interval $\langle 1,2 n\rangle$, a contradiction. Thus $k_{0}$ is odd. If also $k_{1}, k_{2}, \ldots, k_{n}$ would be odd, then there would be $n+1$ odd integers between 1 and $2 n$, a contradiction. Therefore, there exists $m \in\{1,2, \ldots, n\}$ such that $k_{0}, k_{1}, \ldots, k_{m-1}$ are odd and $k_{m}, k_{m+1}, \ldots, k_{n}$ are even. Hence

$$
k_{0}+2(m-1) \leq k_{m-1} \text { and } k_{m} \leq k_{n}-2(n-m) \text {. }
$$


Since furthermore $k_{m-1}<k_{m}$, it follows from (7) that $2 n-1 \leq k_{n}-k_{0}$, and thus $k_{0}=1$ and $k_{n}=2 n$. Putting these values of $k_{0}$ and $k_{n}$ into (7), we obtain $k_{m-1}=2 m-1$ and $k_{m}=2 m$.

By the above, if $b \neq 0$, then $i=1, j=2 n$ and the sequence

$$
\left(k_{0}, k_{1}, k_{2}, \ldots, k_{n-1}, k_{n}\right)
$$

is of the following form:

$$
(\underbrace{1,3, \ldots, 2 m-1}_{\text {odd integers }}, \underbrace{2 m, 2 m+2, \ldots, 2 n}_{\text {even integers }}), \text { for some } m \in\{1,2, \ldots, n\} .
$$

The sequence (8) will be denoted by $\gamma_{m}$ and the set of all such sequences will be denoted by $\mathcal{C}_{n}$, i.e., $\mathcal{C}_{n}=\left\{\gamma_{1}, \gamma_{2}, \ldots, \gamma_{n}\right\}$. For example, $\mathcal{C}_{4}$ consists of the following four sequences:

$$
\gamma_{1}=(1,2,4,6,8), \quad \gamma_{2}=(1,3,4,6,8), \quad \gamma_{3}=(1,3,5,6,8), \quad \gamma_{4}=(1,3,5,7,8) .
$$

To simplify notation, for any $\gamma=\left(k_{0}, k_{1}, \ldots, k_{n-1}, k_{n}\right) \in \mathcal{C}_{n}$, the product $(6)$ will be denoted by $\gamma\left(B_{1}, B_{2}, \ldots, B_{n}\right)$, i.e.,

$$
\gamma\left(B_{1}, B_{2}, \ldots, B_{n}\right)=B_{1}^{\left(k_{0} k_{1}\right)} B_{2}^{\left(k_{1} k_{2}\right)} B_{3}^{\left(k_{2} k_{3}\right)} \cdots B_{n}^{\left(k_{n-1} k_{n}\right)} .
$$

Summarizing, and using the just introduced notation, if $B_{1}, B_{2}, \ldots, B_{n} \in$ $N(R)$, then the $(1,2 n)$ entry of the product $B_{1} B_{2} \cdots B_{n}$ is equal to the sum $\sum_{m=1}^{n} \gamma_{m}\left(B_{1}, B_{2}, \ldots, B_{n}\right)$, and all other entries are equal to 0 , i.e.,

$$
B_{1} B_{2} \cdots B_{n}=\left(\sum_{m=1}^{n} \gamma_{m}\left(B_{1}, B_{2}, \ldots, B_{n}\right)\right) E_{1,2 n} .
$$

Now we are in a position to prove the implication (iii) $\Rightarrow$ (i). Assume $R$ is a reduced commutative ring and $n \geq 2$ is an integer satisfying (iii), i.e., we have $n r=0 \Rightarrow r=0$ for any $r \in R$. To prove (i), we consider an arbitrary polynomial

$$
f=A_{0}+A_{1} x+\cdots+A_{t} x^{t} \in D(R)[x] \text { such that } f^{n}=0,
$$

and we have to show that

$$
A_{s_{1}} A_{s_{2}} \cdots A_{s_{n}}=0 \text { for any } n \text {-tuple }\left(s_{1}, s_{2}, \ldots, s_{n}\right) .
$$

Since $N(R)$ is an ideal of $D(R)$ and the ring $D(R) / N(R)$ is isomorphic to $R$, it follows that $D(R) / N(R)$ is reduced. Hence also the ring

$$
D(R)[x] / N(R)[x] \cong(D(R) / N(R))[x]
$$

is reduced and from $f^{n}=0$ we deduce that $f \in N(R)[x]$. Thus $A_{0}, A_{1}, \ldots, A_{t} \in$ $N(R)$, and (9) implies that to prove (10) it suffices to show that for any $m \in$ $\{1,2, \ldots, n\}$ and $n$-tuple $\left(s_{1}, s_{2}, \ldots, s_{n}\right)$ we have

$$
\gamma_{m}\left(A_{s_{1}}, A_{s_{2}}, \ldots, A_{s_{n}}\right)=0 .
$$


To prove (11), let $\Phi: D(R)[x] \rightarrow D(R[x])$ be the canonical isomorphism and let $F=\Phi(f)$. Since $f \in N(R)[x]$ and $f^{n}=0$, we have $F \in N(R[x])$ and $F^{n}=0$. Hence (9) implies

$$
\sum_{m=1}^{n} \gamma_{m}(\underbrace{F, F, \ldots, F}_{n \text { times }})=0 .
$$

Recall that $\gamma_{m}$ denotes the sequence (8) and thus

$$
\begin{aligned}
& \gamma_{m}(F, F, \ldots, F) \\
= & F^{(1,3)} F^{(3,5)} \ldots F^{(2 m-3,2 m-1)} F^{(2 m-1,2 m)} F^{(2 m, 2 m+2)} \ldots F^{(2 n-2,2 n)} .
\end{aligned}
$$

Note that since $F \in D(R[x])=D_{n}\left(D_{2}(R[x])\right)$, for any odd $i \in\{1,2, \ldots, 2 n\}$ we have $F^{(i, i+1)}=F^{(1,2)}$ and if furthermore $i \leq 2 n-3$, then $F^{(i, i+2)}=F^{(i+1, i+3)}$. Moreover, since $R$ is commutative, the ring $R[x]$ is commutative. Combining all of these with (13), we obtain

$$
\begin{aligned}
\gamma_{m}(F, F, \ldots, F) & =F^{(2,4)} F^{(4,6)} \cdots F^{(2 m-2,2 m)} F^{(1,2)} F^{(2 m, 2 m+2)} \cdots F^{(2 n-2,2 n)} \\
& =F^{(1,2)} F^{(2,4)} F^{(4,6)} \cdots F^{(2 m-2,2 m)} F^{(2 m, 2 m+2)} \cdots F^{(2 n-2,2 n)} \\
& =\gamma_{1}(F, F, \ldots, F) .
\end{aligned}
$$

We have shown that

$$
\gamma_{m}(F, F, \ldots, F)=\gamma_{1}(F, F, \ldots, F) \text { for any } m \in\{1,2, \ldots, n\},
$$

and thus (12) implies

$$
n \cdot \gamma_{1}(F, F, \ldots, F)=0 \text {. }
$$

Since by (iii) we have $n r=0 \Rightarrow r=0$ for any $r \in R$, it follows from (15) that $\gamma_{1}(F, F, \ldots, F)=0$, and thus, by (14), for any $m \in\{1,2, \ldots, n\}$ we have

$$
\gamma_{m}(F, F, \ldots, F)=0 .
$$

We will show that (16) implies (11), which is enough to complete the proof. Let $\gamma_{m}=\left(k_{0}, k_{1}, k_{2}, \ldots, k_{n-1}, k_{n}\right)$ (at this point, the exact form of $\gamma_{m}$ does not matter). From (16) we obtain

$$
F^{\left(k_{0} k_{1}\right)} F^{\left(k_{1} k_{2}\right)} \cdots F^{\left(k_{n-1} k_{n}\right)}=0 .
$$

For any $i \in\{1,2, \ldots, n\}$, set

$$
f_{i}=A_{0}^{\left(k_{i-1} k_{i}\right)}+A_{1}^{\left(k_{i-1} k_{i}\right)} x+\cdots+A_{t}^{\left(k_{i-1} k_{i}\right)} x^{t} \in R[x] .
$$

Since $F=\Phi(f)$, it follows from (17) that

$$
f_{1} f_{2} \cdots f_{n}=0 \text {. }
$$

By assumption the ring $R$ is reduced, so $R$ is Armendariz as well, and thus (18) and Proposition 1.1 imply that for any $n$-tuple $\left(s_{1}, s_{2}, \ldots, s_{n}\right)$ we have

$$
A_{s_{1}}^{\left(k_{0} k_{1}\right)} A_{s_{2}}^{\left(k_{1} k_{2}\right)} \cdots A_{s_{n}}^{\left(k_{n-1} k_{n}\right)}=0,
$$


that is,

$$
\gamma_{m}\left(A_{s_{1}}, A_{s_{2}}, \ldots, A_{s_{n}}\right)=0 \text { for any } m \in\{1,2, \ldots, n\} .
$$

Since $A_{s_{1}}, A_{s_{2}}, \ldots, A_{s_{n}} \in N(R)$, from (9) we obtain

$$
A_{s_{1}} A_{s_{2}} \cdots A_{s_{n}}=0
$$

which is just condition (10). The proof is complete.

Now we are in a position to answer Jeon-Lee-Ryu's problem (5).

Example 3.2. (There exists a nonreduced ring $R$ such that for any $n \geq 2$ the ring $D_{n}(R)$ is $n$-semi-Armendariz.) Let, as usually, $\mathbb{Z}$ denote the ring of integers. The ring $R=D_{2}(\mathbb{Z})$ is not reduced but, by Theorem 3.1, for any $n \geq 2$ the ring $D_{n}(R)$ is $n$-semi-Armendariz.

Remark 3.3. Using the same arguments as in the proof of Theorem 3.1 one can prove the following generalization of Theorem 3.1.

Let $S$ be a reduced subring of a commutative Armendariz ring $R$ and let $n \geq 2$ be an integer. Then the following conditions are equivalent:

(i) The ring $D_{n}\left(D_{2}(S, R)\right)$ is n-semi-Armendariz;

(ii) The ring $\left(D_{2}(S, R)[x]\right) /\left(x^{n}\right)$ is n-semi-Armendariz, where $\left(x^{n}\right)$ denotes the ideal of $D_{2}(S, R)[x]$ generated by $x^{n}$;

(iii) $n r=0 \Rightarrow r=0$ for any $r \in R$.

Let $R$ be a ring and let $I$ be a proper ideal of $R$. Assume $R / I$ and $I$ are $n$ semi-Armendariz, where $I$ is considered as an $n$-semi-Amendariz ring without unity. One could conjecture that in this case $R$ has to be $n$-semi-Armendariz. However, this is not the case as shown in [7, Example 2.2]. Below we use Theorem 3.1 to construct another example of a ring $R$ with an ideal $I$ such that $R / I$ and $I$ are $n$-semi-Armendariz but $R$ is not $n$-semi-Armendariz.

Example 3.4. (For any $n \geq 2$ there exist a ring $R$ and an ideal $I$ of $R$ such that $I$ and $R / I$ are $n$-semi-Armendariz, but $R$ is not $n$-semi-Armendariz.) Let $n=p_{1}^{\alpha_{1}} p_{2}^{\alpha_{2}} \cdots p_{k}^{\alpha_{k}}$ be the prime factorization of $n$ and let $m=p_{1} p_{2} \cdots p_{k}$. Note that the ring $\mathbb{Z}_{m}$ of integers modulo $m$ is reduced. Set $R=\left(D_{2}\left(\mathbb{Z}_{m}\right)[x]\right) /\left(x^{n}\right)$ and $I=\left(x^{n-1}\right) /\left(x^{n}\right)$. Then $I$ is an ideal of $R$ with $I^{2}=0$ and thus $I$ is $n$-semiArmendariz. Furthermore, the $\operatorname{ring} R / I \cong\left(D_{2}\left(\mathbb{Z}_{m}\right)[x]\right) /\left(x^{n-1}\right)$ is isomorphic to a subring of $U_{n-1}\left(D_{2}\left(\mathbb{Z}_{m}\right)\right.$ ) (see the proof of the implication (i) $\Rightarrow$ (ii) in Theorem 3.1). Since $N=N_{2}\left(\mathbb{Z}_{m}\right)$ is an ideal of $D_{2}\left(\mathbb{Z}_{m}\right)$ such that the ring $D_{2}\left(\mathbb{Z}_{m}\right) / N \cong \mathbb{Z}_{m}$ is reduced and $N^{2}=0$, Proposition 2.4 implies that the ring $U_{n-1}\left(D_{2}\left(\mathbb{Z}_{m}\right)\right)$ is $n$-semi-Armendariz, and thus the ring $R / I$ is $n$ semi-Armendariz as well. Finally note that by Theorem 3.1 the ring $R$ is not $n$-semi-Armendariz.

Recall that a ring $R$ is said to be abelian if every idempotent of $R$ is central. In $[7$, Example 2.4(1)] it was shown that an abelian prime ring need not be semi-Armendariz. Bellow we show the same, constructing a simpler example than this in [7]. 
Example 3.5. (An abelian prime ring need not be semi-Armendariz.) Let $S$ be a domain and let $n \geq 2$. Set $R=D_{n+2}(S)$. Then by [5, Lemma 2] the ring $R$ is abelian. To see that $R$ is prime, consider any non-zero matrices $A, B \in R$. Let $a_{i j}$ and $b_{k l}$ be any nonzero entries of $A$ and $B$, respectively. Then the $(i, l)$ entry of $A E_{j k} B$ is equal to $a_{i j} b_{k l} \neq 0$. Thus $A R B \neq 0$, proving that $R$ is prime. Finally, Lemma 2.9 implies that $R$ is not $n$-semi-Armendariz and thus $R$ is not semi-Armendariz.

We close the paper with an example of a commutative ring which is not semi-Armendariz (cf. [7, Example 2.4(2)]).

Example 3.6. (A commutative ring need not be semi-Armendariz.) Obviously, the ring $R=D_{2}\left(D_{2}\left(\mathbb{Z}_{2}\right)\right)$ is commutative. However, $R$ is not 2-semiArmendariz by Theorem 3.1 and thus $R$ is not semi-Armendariz.

Acknowledgments. The research of Ryszard Mazurek was supported by the Polish National Center of Science Grant No DEC-2011/03/B/ST1/04893, carried out at the University of Warsaw.

\section{References}

[1] D. D. Anderson and V. Camillo, Armendariz rings and Gaussian rings, Comm. Algebra 26 (1998), no. 7, 2265-2272.

[2] E. P. Armendariz, A note on extensions of Baer and P.P.-rings, J. Austral. Math. Soc. 18 (1974), 470-473.

[3] B. J. Gardner and R. Wiegandt, Radical theory of rings, Monographs and Textbooks in Pure and Applied Mathematics, vol. 261, Marcel Dekker, Inc., New York, 2004.

[4] Y. Hirano, On annihilator ideals of a polynomial ring over a noncommutative ring, J. Pure Appl. Algebra 168 (2002), no. 1, 45-52.

[5] C. Huh, H. K. Kim, and Y. Lee, p.p. rings and generalized p.p. rings, J. Pure Appl. Algebra 167 (2002), no. 1, 37-52.

[6] C. Huh, Y. Lee, and A. Smoktunowicz, Armendariz rings and semicommutative rings, Comm. Algebra 30 (2002), no. 2, 751-761.

[7] Y. C. Jeon, Y. Lee, and S. J. Ryu, A structure on coefficients of nilpotent polynomials, J. Korean Math. Soc. 47 (2010), no. 4, 719-733.

[8] N. K. Kim and Y. Lee, Armendariz rings and reduced rings, J. Algebra 223 (2000), no. $2,477-488$.

[9] N. K. Kim, K. H. Lee, and Y. Lee, Power series rings satisfying a zero divisor property, Comm. Algebra 34 (2006), no. 6, 2205-2218.

[10] T. Y. Lam, A first Course in Noncommutative Rings, Graduate Texts in Math., vol. 131, Springer-Verlag, Berlin-Heidelberg-New York 1991.

[11] T.-K. Lee and T.-L. Wong, On Armendariz rings, Houston J. Math. 29 (2003), no. 3, 583-593.

[12] Z. Liu and R. Zhao, On weak Armendariz rings, Comm. Algebra 34 (2006), no. 7, 2607-2616.

[13] G. Marks, R. Mazurek, and M. Ziembowski, A unified approach to various generalizations of Armendariz rings, Bull. Aust. Math. Soc. 81 (2010), no. 3, 361-397.

[14] R. Mazurek and M. Ziembowski, Right Gaussian rings and skew power series rings, J. Algebra 330 (2011), no. 1, 130-146.

[15] - On a characterization of distributive rings via saturations and its applications to Armendariz and Gaussian rings, Rev. Mat. Iberoam. 30 (2014), no. 3, 1073-1088. 
KAMIL KozŁOWSKI

Faculty of Computer Science

Bialystok University of TeChNOlogy

Wiejska 45A, 15-351 Biąystok, Poland

E-mail address: kam.kozlowski93@gmail.com

RYSZARD MAZUREK

Faculty of Computer Science

Bialystok University of Technology

Wiejska 45A, 15-351 Biąystok, Poland

E-mail address: r.mazurek@pb.edu.pl 